\title{
The effect of duration on the relative detectability of brief tonal bursts and gaps in the tone
}

\author{
SIMON KEMP \\ University of Canterbury, Christchurch, New Zealand
}

(K. T. Strongman, Sponsor)

\begin{abstract}
It is known that a burst of tone in noise is easier to detect than an equal duration gap in the same tone. A theory of temporal summation (Irwin \& Kemp, 1976) predicts that the difference in detectability should decrease if the duration of the events is increased. A forced-choice experiment was performed with two durations ( 25 and $200 \mathrm{msec}$ ) and $\mathrm{E} / \mathrm{n}_{0}$ held constant at 15 for bursts and 30 for gaps. As predicted, results showed shorter duration events to differ more in detectability than longer ones.
\end{abstract}

It has been shown that a brief gap in a continuous tone is less readily detected than a brief tone burst (Gilliom \& Mills, 1976; Irwin \& Kemp, 1976; Schulman, 1971). If the durations of burst and gap are equated and the frequency of the tone and level of masking noise are kept constant, then the level of the tone surrounding the gap must be about 3-4 dB greater than that of the tone burst in order to obtain equal detectability.

The phenomenon by which the detectability or loudness of a brief tone burst increases with its duration is known as temporal summation and is generally believed to arise from the occurrence of an active summation process in the auditory system (e.g., Garner \& Miller, 1947; Zwislocki, 1969). It is attractive and parsimonious to hypothesize that the difference in detectability between bursts and gaps also arises from a summation process, and in fact Irwin and Kemp (1976) have proposed a model of temporal summation that predicts reasonably well existing data on the detection of bursts and gaps. This paper examines and evaluates an experimental prediction that can be derived from their model.

The model, which is illustrated in Figure 1, assumes a running average, expressed by the integral

$$
\mathrm{y}(\mathrm{t})=\int_{-\infty}^{\infty} \mathrm{x}(\tau) \mathrm{w}(\mathrm{t}-\tau) \mathrm{d} \tau,
$$

to be performed at some point in the auditory system. The weighting function is given by $\mathrm{w}(\mathrm{t}-\tau)=\mathrm{e}^{-\alpha(\mathrm{t}-\tau)}$, where $\alpha$ is a time constant, equal to $5 \mathrm{~s}^{-1}$. The input to the running average is assumed to take the form

I am grateful to R. J. Irwin for useful discussion on an earlier version of the paper. The research was partially funded by the New Zealand University Grants Committee, and the experiment performed at the University of Auckland. My mailing address is: Department of Psychology, University of Canterbury, Christchurch 1, New Zealand.

$$
\begin{aligned}
x(t)= & A+B e^{-\gamma t} \text { when the tone is turned on; } \\
& x(t)=0 \text { when the tone is off. }
\end{aligned}
$$

This form of $\mathrm{x}(\mathrm{t})$ mimics the response of the auditory nerve to a constant tone. The transient response, $\mathrm{Be}^{-\gamma \mathrm{t}}$,

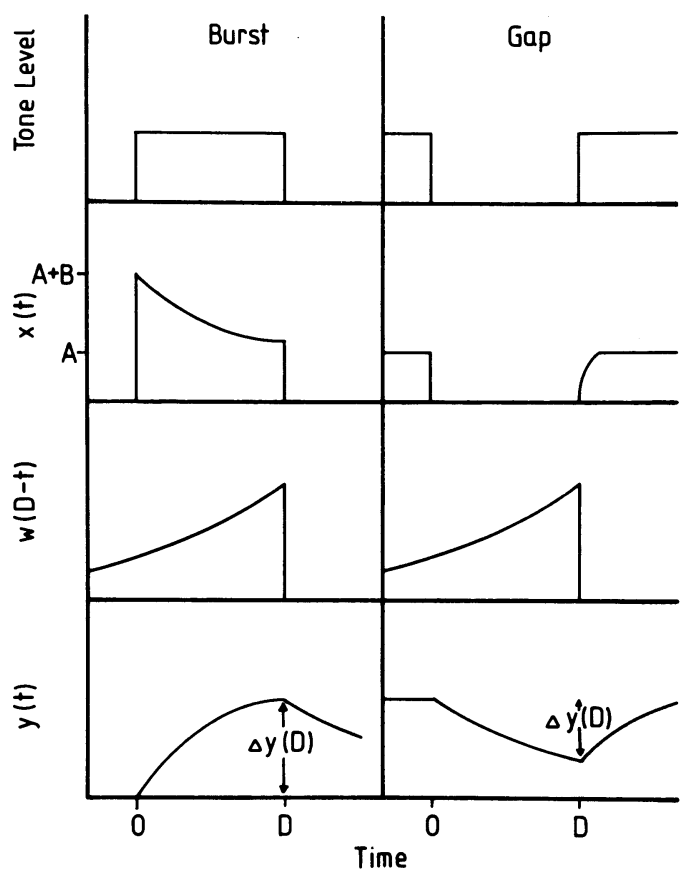

Figure 1. Illustration of Irwin and Kemp's (1976) model of why bursts of a tone are more readily detected than gaps in it. The top line shows the tone levels pertaining to the two kinds of event as a function of time. The second line shows the resultant $x(t)$ for the events (see Equation 2), and the third line shows the (common) weighting function. The final line shows the resulting $y(t)$ function after convolution. Note that $\Delta y(D)$ for the burst is bigger than $\Delta y(D)$ for the gap (Equations 3 and 4); hence the burst should be more readily detected. 
is assumed to occur only to tone bursts and not as the tone is switched on again after a brief gap. When the output of the running average, $y(t)$, is evaluated at the end of a tone burst of duration, $D$, the maximum change in $y(t)$, $\Delta y(D)$, is given by

$$
\Delta y(D)=\frac{A}{\alpha}\left(1-e^{-\alpha D}\right)+\left(\frac{B}{\gamma-\alpha}\right)\left(e^{-\alpha D}-e^{-\gamma D}\right) .
$$

When the running average is evaluated at the end of a tone gap of duration, $D$, the maximum change in $y(t)$, $\Delta y(D)$, is given by

$$
\Delta y(D)=\frac{A}{\alpha}\left(1-e^{-\alpha D}\right) .
$$

The detectability of a burst or gap is assumed to be monotonically functionally related to $\Delta y(D)$. Differing detectability of bursts and gaps then arises because of the difference betwen Equations 3 and 4 and because, for any positive constants, $\mathrm{B}, \lambda$, and $\alpha$,

$$
\left(\frac{B}{\gamma-\alpha}\right)\left(e^{-\alpha D}-e^{-\gamma D}\right)
$$

is greater than or equal to zero. Essentially, the model's prediction of a difference in detectability between bursts and gaps arises because of the transient response to the onset of the burst. The difference between Equations 3 and 4 arises mathematically from the $\mathrm{Be}^{-\gamma t}$ term in the burst input function, $\mathrm{x}(\mathrm{t})=\mathrm{A}+\mathrm{Be}^{-\gamma \mathrm{t}}$. Clearly with increasing time, this transient response becomes less important because the relative contribution made by $\mathrm{Be}^{-\gamma \mathrm{t}}$ to $x(t)$ decreases with $t$. Consider two bursts of unequal duration. If the burst is short, the contribution of the transient response will be relatively large; if the burst is long, the contribution will be relatively small. The effect is enhanced when the weighting function is considered because, for the longer burst, the recent input, when the contribution of the transient is relatively least, is maximally weighted, and the $\mathrm{x}(\mathrm{t})$ response at sound onset is weighted least. The implication of this reasoning is that one should expect the difference in detectability between gaps and bursts to decrease if the duration common to both types of event is increased.

Irwin and Kemp's data show some slight indication of this effect in that, at a duration of $200 \mathrm{msec}$, gaps with a signal-power to noise-power-per-cycle ratio $\left(\mathrm{P} / \mathrm{N}_{\mathrm{o}}\right)$ of $300 \mathrm{sec}^{-1}$ were more readily detected on average than bursts with $P / N_{o}$ equal to $150 \mathrm{sec}^{-1}$, but this result was reversed for briefer durations (Irwin \& Kemp, 1976, Figure 2). Most of the data, however, do not test this expectation: Irwin and Kemp generally kept $\mathrm{P} / \mathrm{N}_{\mathrm{o}}$ constant and varied duration. Under these circumstances, the detectability of both bursts and gaps increases with duration, and differences in their relative detectabilities are ob- scured. The present experiment sought to make differences in detectability more noticeable by holding the signal energy to noise-power-per-cycle ratio $\left(E / N_{o}\right)$ of the bursts constant, because, in this case, the detectability of the tone bursts should remain approximately constant (Garner \& Miller, 1947; Green, Birdsall, \& Tanner, 1957). $E / N_{o}$ for the gaps, defined here as the tonal energy missing in the gap as a ratio of the noise power per cycle, was also held constant, and gap duration was varied. The level of the tone surrounding the gaps was set $3 \mathrm{~dB}$ above the level in the comparable bursts to obtain roughly comparable detectabilities of the two types of event.

\section{METHOD}

\section{Subjects}

Six subjects with normal hearing served in the experiment.

\section{Apparatus}

The event to be detected was either a tone burst or a gap in a tone. A 1-kHz tone was generated and switched on and off with a rise-decay time of $1.7 \mathrm{msec}$. After attenuation, the tone signal was electronically mixed with a continuous white noise whose $3 \mathrm{~dB}$ bandpass (Krohn-Hite, model 3550R) was set between 100 and $5000 \mathrm{~Hz}$ (giving an equivalent rectangular bandwidth of $5028 \mathrm{~Hz}$ ) and whose level was set to $56 \mathrm{~dB}$ SPL.

The sound was presented monaurally by means of a TDH-39 earphone. Subjects sat in a sound attenuating chamber and responded by pressing buttons. The timing of the experiment was controlled by a microprocessor.

\section{Procedure}

Subjects were required to detect events using the two-alternative forcedchoice procedure. In each trial, the event to be detected occurred with equal probability in one of two intervals, marked for the subject by lights. Trials were given in blocks of 100 with four blocks, each of a different experimental condition, being given in each session; within each block the stimulus parameters were unchanged. Subjects listened to two types of events, bursts and gaps that lasted 25 or $200 \mathrm{msec}$. E/ $\mathrm{N}_{\text {o }}$ was maintained at $\mathbf{1 5}$ for the burst and $\mathbf{3 0}$ for the gap conditions. There were four experimental and one or two practice sessions for each subject; this yielded 400 trials per experimental condition per subject.

\section{RESULTS AND DISCUSSION}

In Table 1, the percentage of correct responses is given for each subject and experimental condition. Analysis of variance on the results showed a significant main effect of duration $[F(1,5)=51.6, p<0.05]$, but not of event type $[F(1,5)=1.30$, n.s.]. These main effects are not of particular consequence here in view of the different $\mathrm{E} / \mathrm{N}_{\mathrm{o}}$ for burst and gap events.

\section{Table 1}

Percentage of Correct Responses for Each Subject and Experimental Condition Averaged over 400 Trials

\begin{tabular}{ccccc}
\hline & \multicolumn{2}{c}{$25 \mathrm{msec}$} & \multicolumn{2}{c}{$200 \mathrm{msec}$} \\
Subjects & Burst & Gap & Burst & Gap \\
\hline 1 & 91.5 & 85.8 & 75.0 & 89.0 \\
2 & 89.0 & 88.0 & 78.0 & 78.8 \\
3 & 92.8 & 88.5 & 85.3 & 81.0 \\
4 & 91.3 & 85.8 & 82.5 & 84.8 \\
5 & 81.0 & 55.8 & 64.5 & 53.0 \\
6 & 81.3 & 74.3 & 71.5 & 75.8 \\
Average & 87.8 & 76.1 & 79.7 & 77.0 \\
\hline
\end{tabular}


The important result is a significant interaction between event type and duration $[\mathrm{F}(1,5)=8.81, \mathrm{p}<0.05]$. For the shorter duration, bursts were much more readily detected than gaps; when the duration was $200 \mathrm{msec}$, however, the difference in detectability was greatly reduced.

The result of the experiment clearly confirms the prediction derived in the introduction. Increasing the duration of both burst and gap events reduces the difference in detectability between them, as was predicted by Irwin and Kemp's (1976) model. Thus the result can be seen as supporting the existence of processes of temporal summation in the auditory system.

\section{REFERENCES}

Garner, W. R., \& Miller, G. A. (1947). The masked threshold of pure tones as a function of duration. Journal of Experimental Psychology, 37, 294-303.
Gilliom, J. D., \& Mills, W. M. (1976). Gap detection: Two channel detection of the missing event. Journal of the Acoustical Society of America, 60, 395-404.

Green, D. M., Birdsall, T. G., \& Tanner, W. P., JR. (1957). Signal detection as a function of signal intensity and duration. Journal of the Acoustical Society of America, 29, 523-531.

IRWIN, R. J., \& KeMP, S. (1976). Temporal summation and decay in hearing. Journal of the Acoustical Society of America, 59, 920-925.

PenNer, M. J. (1975). Persistence and integration: Two consequences of a sliding integrator. Perception \& Psychophysics, 18, 114-120.

Schulman, A. I. (1971). Detectability of the deletion of a tone from a tone-plus-noise background. Perception \& Psychophysics, 9, 496-498.

ZWISLOCKI, J. J. (1969). Temporal summation of loudness: An analysis. Journal of the Acoustical Society of America, 46, 431-441.

(Manuscript received for publication April 13, 1985.) 